УДК 519.218.2

\title{
On Application of Slowly Varying Functions with Remainder in the Theory of Galton-Watson Branching Process
}

\author{
Azam A. Imomov* \\ State Testing Center under the Cabinet of Ministers of the Republic of Uzbekistan \\ 12, Bogishamol st., 100202, Tashkent \\ Karshi State University \\ 17, Kuchabag st., Karshi city, 180100 \\ Uzbekistan \\ Erkin E. Tukhtaev ${ }^{\dagger}$ \\ Karshi State University \\ 17, Kuchabag st., Karshi city, 180100 \\ Uzbekistan
}

Received 27.06.2018, received in revised form 17.08.2018, accepted 07.10.2018

We investigate an application of slowly varying functions (in sense of Karamata) in the theory of GaltonWatson branching processes. Consider the critical case so that the generating function of the per-capita offspring distribution has the infinite second moment, but its tail is regularly varying with remainder. We improve the Basic Lemma of the theory of critical Galton-Watson branching processes and refine some well-known limit results.

Keywords: Galton-Watson branching process, slowly varying functions, generating functions.

DOI: 10.17516/1997-1397-2019-12-1-51-57.

\section{Introduction and preliminaries}

A conception of slow variation (or more general - regular variation) was initiated first by Jovan Karamata in [7,8]. Zolotarev [15] one of the first demonstrated an encouraging perspective of application of the conception of slow variation in probability theory, in particular in the theory of stochastic branching processes. Afterwards Slack [13,14] and Seneta [9], [10,12] prove principally new limit theorems for branching processes using slowly varying (SV) functions. Remind that real-valued, positive and measurable function $\ell(x)$ is said to be $\mathrm{SV}$ at infinity in sense of Karamata if $\ell(\lambda x) / \ell(x) \rightarrow 1$ as $x \rightarrow \infty$ for each $\lambda>0$. A function $\mathrm{V}(x)$ is said to be regularly varying at infinity with index of regular variation $\rho \in \mathbb{R}_{+}$if it in the form $\mathrm{V}(x)=x^{\rho} \ell(x)$, where $\ell(x)$ is $\mathrm{SV}$ at infinity. We refer the reader to [1,3] and [11] for more information.

Let $F(s)=\sum_{j \in \mathbb{N}_{0}} p_{j} s^{j}$ denote an offspring probability generating function (PGF) of GaltonWatson (GW) branching process, where $\mathbb{N}_{0}=\{0\} \cup \mathbb{N}$ and $\mathbb{N}=\{1,2, \ldots\}$. Supposing that $p_{0}>0$ we consider the case when the mean per-capita offspring number $\sum_{j \in \mathbb{N}} j p_{j}=1$, that is the

\footnotetext{
*imomov azam@mail.ru

†erkin.tuxtayev@mail.ru

(c) Siberian Federal University. All rights reserved
} 
process is critical, see [2]. Moreover we assume that PGF $F(s)$ for $0 \leqslant s<1$ has the following representation:

$$
F(s)=s+(1-s)^{1+\nu} \mathcal{L}\left(\frac{1}{1-s}\right),
$$

where $0<\nu \leqslant 1$ and $\mathcal{L}(t)$ is SV at infinity. By the criticality of our process the condition (1) implies that the second moment $F^{\prime \prime}(1-)=\infty$. This includes the case $F^{\prime \prime}(1-)<\infty$ when $\nu=1$ and $\mathcal{L}(t) \rightarrow F^{\prime \prime}(1-) / 2$ as $t \rightarrow \infty$.

Let $Z_{n}$ be the population size in $n$-th generation. Process evolution is characterized by transition probabilities $P_{i j}(n):=\mathbb{P}\left\{Z_{n}=j \mid Z_{0}=i\right\}$. In this interpretation $p_{j}=\mathbb{P}\left\{Z_{1}=j\right\}$ provided that $\mathbb{P}\left\{Z_{0}=1\right\}=1$. A PGF

$$
F_{n}(s)=\sum_{j \in \mathbb{N}_{0}} P_{1 j}(n) s^{j}
$$

is the $n$-fold iteration of $F(s)$, see [2]. Further by the symbol $\mathcal{H}=\min \left\{n: Z_{n}=0\right\}$ we denote a time of extinction of GW process. Write $R_{n}(s):=1-F_{n}(s)$ and needless to say $Q_{n}:=$ $\mathbb{P}\{\mathcal{H}>n\}=R_{n}(0)$.

The following theorem is known.

Theorem S [14]. If the condition (1) holds then

$$
\lim _{n \rightarrow \infty} \mathbb{P}\left\{Q_{n} Z_{n} \leqslant x \mid \mathcal{H}>n\right\}=G(x),
$$

where $G(x)$ has the Laplace transform

$$
\Psi(\theta)=1-\left(1+\theta^{-\nu}\right)^{-1 / \nu}
$$

By arguments of Slack [14] one can be shown that if the condition (1) holds then

$$
Q_{n}^{\nu} \mathcal{L}\left(\frac{1}{Q_{n}}\right) \sim \frac{1}{\nu n} \quad \text { as } n \rightarrow \infty .
$$

Slack [14] also has shown that

$$
\mathcal{U}_{n}(s):=\frac{F_{n}(s)-F_{n}(0)}{F_{n}(0)-F_{n-1}(0)} \longrightarrow U(s) \text { as } n \rightarrow \infty,
$$

for $0 \leqslant s<1$, where $U(F(s))=U(s)+1$ and

$$
U(s)=\frac{1+o(1)}{\nu(1-s)^{\nu} \mathcal{L}(1 /(1-s))} \quad \text { as } \quad s \uparrow 1 .
$$

Combining (1), (2) and (3) we have

$$
\mathcal{U}_{n}(s) \sim U_{n}(s):=\left[1-\frac{R_{n}(s)}{Q_{n}}\right] \nu n \quad \text { as } n \rightarrow \infty .
$$

So we have proved the following lemma as a generalization of the assertion (2) for all $s \in[0,1)$.

Lemma 1.1. If the condition (1) holds then

$$
R_{n}(s)=\frac{\mathcal{N}(n)}{(\nu n)^{1 / \nu}} \cdot\left[1-\frac{U_{n}(s)}{\nu n}\right],
$$

where the function $\mathcal{N}(x)$ is $S V$ at infinity and

$$
\mathcal{N}(n) \cdot \mathcal{L}^{1 / \nu}\left(\frac{(\nu n)^{1 / \nu}}{\mathcal{N}(n)}\right) \longrightarrow 1 \text { as } n \rightarrow \infty,
$$

and the function $U_{n}(s)$ enjoys following properties: 
(i) $U_{n}(s)=U(s)(1+o(1))$ as $n \rightarrow \infty$,

(ii) $\lim _{s \uparrow 1} U_{n}(s)=\nu n$ for each fixed $n \in \mathbb{N}$,

(iii) $U_{n}(0)=0$ for each fixed $n \in \mathbb{N}$.

We obtain the Lemma 1.1 by more simple proof rather than as shown in [6]. This lemma is called the Basic Lemma of the theory of critical GW branching process. The following lemma established in [6] is differential analogue of Lemma 1.1

Lemma 1.2. If the condition (1) holds then

$$
\frac{\partial R_{n}(s)}{\partial s}=-\left(\frac{R_{n}(s)}{1-s}\right)^{1+\nu} \frac{\mathcal{L}\left(1 / R_{n}(s)\right)}{\mathcal{L}(1 /(1-s))} .
$$

Since $\mathcal{L}(x)$ is SV-function we can write

$$
\frac{\mathcal{L}(\lambda x)}{\mathcal{L}(x)}=1+\alpha(x)
$$

for each $\lambda>0$, where $\alpha(x) \rightarrow 0$ as $x \rightarrow \infty$. Henceforth we suppose that some positive function $g(x)$ is given so that $g(x) \rightarrow 0$ and $\alpha(x)=o(g(x))$ as $x \rightarrow \infty$. In this case $\mathcal{L}(x)$ is called SV with remainder, see [3, p. 185, condition SR3].

We devote this paper to improvement of the Lemma 1.1 provided that the condition (4) holds with given $\alpha(x)$. Subsequently of this we will improve the Lemma 1.2 and define a speed rate in some well-known limit theorems from the theory of critical GW branching process.

\section{Improvement of the Basic Lemma and results}

Everywhere in this section we suppose the condition (4) holds. Write

$$
\Lambda(y):=\frac{F(1-y)-(1-y)}{y}=y^{\nu} \mathcal{L}\left(\frac{1}{y}\right) .
$$

Note that the function $y \Lambda(y)$ is positive and tends to zero and has a monotone derivative for $y \in(0,1]$ so that $y \Lambda^{\prime}(y) / \Lambda(y) \rightarrow \nu$ as $y \downarrow 0$, see [3, p. 401]. Hence we can write

$$
\frac{y \Lambda^{\prime}(y)}{\Lambda(y)}=\nu+\delta(y)
$$

where $\delta(y)$ is continuous and $\delta(y) \rightarrow 0$ as $y \downarrow 0$. Integrating this equality we obtain

$$
\Lambda(y)=p_{0} y^{\nu} \exp \int_{1}^{y} \frac{\delta(u)}{u} d u .
$$

We have considered that $\Lambda(1)=\mathcal{L}(1)=p_{0}$ in last step. Therefore we have

$$
\mathcal{L}\left(\frac{1}{y}\right)=p_{0} \exp \int_{1}^{y} \frac{\delta(u)}{u} d u \text {. }
$$

Changing variables as $u=1 / t$ in integrand gives

$$
\mathcal{L}(x)=p_{0} \exp \int_{1}^{x} \frac{\varepsilon(t)}{t} d t,
$$

where $\varepsilon(t)$ is continuous and $\varepsilon(t) \rightarrow 0$ as $t \rightarrow \infty$.

It follows from (5) and (4) that 


$$
\frac{\mathcal{L}(\lambda x)}{\mathcal{L}(x)}=\exp \int_{x}^{\lambda x} \frac{\varepsilon(t)}{t} d t=1+\alpha(x) \quad \text { as } \quad x \rightarrow \infty
$$

for each $\lambda>0$. Hereof

$$
\int_{x}^{\lambda x} \frac{\varepsilon(t)}{t} d t=\ln [1+\alpha(x)]=\alpha(x)+\mathcal{O}\left(\alpha^{2}(x)\right) \quad \text { as } \quad x \rightarrow \infty .
$$

Using the mean value theorem in the left-hand side of this equality we can be convinced that

$$
\varepsilon(x)=\mathcal{O}(\alpha(x)) \quad \text { as } x \rightarrow \infty
$$

Further we will consider a case when

$$
\alpha(x)=o\left(\frac{\mathcal{L}(x)}{x^{\nu}}\right) \quad \text { as } x \rightarrow \infty .
$$

Denote

$$
\phi(y):=1-F(1-y)=y-y \Lambda(y) .
$$

In pursuance of reasoning from [14] we obtain the following asymptotic relation:

$$
\frac{1}{\Lambda(\phi(y))}-\frac{1}{\Lambda(y)}=\nu+\delta(y)
$$

where $\delta(y)$ is continuous function so that $\delta(y) \rightarrow 0$ as $y \downarrow 0$, see also [3, p. 401].

Further discussions allow us to estimate the tail-part $\delta(y)$ in (8). At first we will prove the following lemma.

Lemma 2.1. Let conditions (1), (4) and (7) hold. Then

$$
\mathcal{L}\left(\frac{1}{\phi(y)}\right)=\mathcal{L}\left(\frac{1}{y}\right)(1+o(\Lambda(y))) \quad \text { as } y \downarrow 0 .
$$

Proof. Since the function $\mathcal{L}(x)=x^{\nu} \Lambda(1 / x)$ is differentiable, by virtue of the mean value theorem we have

$$
\mathcal{L}\left(\frac{x}{1-\Lambda}\right)-\mathcal{L}(x)=\mathcal{L}^{\prime}\left(\frac{1-\theta \Lambda}{1-\Lambda} x\right) \cdot \frac{x \Lambda}{1-\Lambda},
$$

where $\Lambda:=\Lambda(1 / x)$ and $0<\theta<1$. From integral representation (5) and considering (6) it follows that

$$
\mathcal{L}^{\prime}(u)=\mathcal{L}(u) \frac{\varepsilon(u)}{u}=o\left(\frac{\mathcal{L}^{2}(u)}{u^{1+\nu}}\right) \quad \text { as } \quad u \rightarrow \infty .
$$

Denote $u=(1-\theta \Lambda) x /(1-\Lambda)$. Since $\Lambda(1 / x) \rightarrow 0$ then $u \sim x$ and $\mathcal{L}(u) \sim \mathcal{L}(x)$ as $x \rightarrow \infty$. Therefore using (11) in the equality (10) and after some elementary transformations the assertion (9) readily follows. The lemma is proved.

Lemma 2.2. Let conditions (1), (4) and (7) hold. Then

$$
\frac{1}{\Lambda(\phi(y))}-\frac{1}{\Lambda(y)}=\nu+\frac{\nu(\nu+1)}{2} \Lambda(y)+\gamma(y)
$$

where $\gamma(y)=o(\Lambda(y))$ as $y \downarrow 0$.

Proof. Write

$$
K(y):=\frac{1}{\Lambda(\phi(y))}-\frac{1}{\Lambda(y)}=\frac{\mathcal{L}\left(\frac{1}{y}\right)-(1-\Lambda(y))^{\nu} \mathcal{L}\left(\frac{1}{\phi(y)}\right)}{\Lambda(y) \cdot(1-\Lambda(y))^{\nu} \mathcal{L}\left(\frac{1}{\phi(y)}\right)}
$$


Taking into consideration (9) the last relation becomes

$$
K(y)=\frac{1-(1-\Lambda(y))^{\nu}}{\Lambda(y)(1-\Lambda(y))^{\nu}}(1+o(\Lambda(y))) \quad \text { as } \quad y \downarrow 0 .
$$

By the Taylor expansion the head part of (13)

$$
\frac{1-(1-\Lambda(y))^{\nu}}{\Lambda(y)(1-\Lambda(y))^{\nu}}=\nu+\frac{\nu(\nu+1)}{2} \Lambda(y)+\mathcal{O}\left(\Lambda^{2}(y)\right) \quad \text { as } \quad y \downarrow 0 .
$$

From here and (13) the formula (12) now easily follows. The lemma is proved.

The following assertion is improved analogy of the Basic Lemma.

Lemma 2.3. Let conditions (1), (4) and (7) hold. Then

$$
\frac{1}{\Lambda\left(R_{n}(s)\right)}-\frac{1}{\Lambda(1-s)}=\nu n+\frac{1+\nu}{2} \cdot \ln (1+\nu n \Lambda(1-s))+\rho_{n}(s)
$$

where $\rho_{n}(s)=o(\ln n)+\sigma_{n}(s)$ and, $\sigma_{n}(s)$ is bounded uniformly for $s \in[0,1)$ and converges to a limit $\sigma(s)$ as $n \rightarrow \infty$ which is a bounded function of $s \in[0,1)$.

Proof. Note that $R_{k+1}(s)=\phi\left(R_{k}(s)\right)$. It is known that $R_{k}(s) \rightarrow 0$ as $k \rightarrow \infty$ uniformly for $s \in[0,1)$, see $[2$, p. 6$]$. Therefore putting $y=R_{k}(s)$ it follows from (12) that

$$
\frac{1}{\Lambda\left(R_{k+1}(s)\right)}-\frac{1}{\Lambda\left(R_{k}(s)\right)}=\nu+\frac{\nu(\nu+1)}{2} \Lambda\left(R_{k}(s)\right)+\gamma\left(R_{k}(s)\right),
$$

where $\gamma(y)=o(\Lambda(y))$ as $y \downarrow 0$. Summing both sides of last equality on $k$ from 1 to $n$ we obtain

$$
\frac{1}{\Lambda\left(R_{n}(s)\right)}-\frac{1}{\Lambda\left(R_{0}(s)\right)}=\nu n+\frac{\nu(\nu+1)}{2} \sum_{k=0}^{n-1} \Lambda\left(R_{k}(s)\right)+\sum_{k=0}^{n-1} \gamma_{k}(s)
$$

where $\gamma_{k}(s)=o\left(\Lambda\left(R_{k}(s)\right)\right)$. Since $\Lambda\left(R_{n}(s)\right) \rightarrow 0$ uniformly for $s \in[0,1)$ each of the last two sums on the right-hand side of $(15)$ is $o(n)$ as $n \rightarrow \infty$. So that considering $R_{0}(s)=1-s$, we have

$$
\frac{1}{\Lambda\left(R_{n}(s)\right)}-\frac{1}{\Lambda(1-s)} \sim \nu n \quad \text { as } n \rightarrow \infty
$$

Thus and so $\nu n \Lambda\left(R_{n}(s)\right) \rightarrow 1$ uniformly for $s \in[0,1)$ as $n \rightarrow \infty$. Hence

$$
\sum_{k=0}^{n-1} \Lambda\left(R_{k}(s)\right)=\mathcal{O}(\ln n)
$$

and $\sum_{k=0}^{n-1} \gamma_{k}(s)=o(\ln n)$ as $n \rightarrow \infty$. Thus we obtain

$$
\frac{1}{\Lambda\left(R_{n}(s)\right)}-\frac{1}{\Lambda(1-s)}=\nu n+\mathcal{O}(\ln n) \quad \text { as } n \rightarrow \infty
$$

Next, using (16), we have 


$$
\begin{aligned}
u_{k}(s):=\Lambda\left(R_{k}(s)\right)-\frac{1}{\nu k+\Lambda^{-1}(1-s)} & =\frac{\mathcal{O}(\ln k)}{\left(\nu k+\Lambda^{-1}(1-s)+\mathcal{O}(\ln k)\right)\left(\nu k+\Lambda^{-1}(1-s)\right)}= \\
& =\frac{\mathcal{O}(\ln k)}{\left(\nu k+\Lambda^{-1}(1-s)\right)^{2}+\mathcal{O}\left(\left(k+\Lambda^{-1}(1-s)\right) \ln k\right)}
\end{aligned}
$$

Since $0 \leqslant s<1$, the right-hand side of last equality is $\mathcal{O}\left(\ln k / k^{2}\right)$. Hence it follows that $\sum_{k \in \mathbb{N}_{0}}\left|u_{k}(s)\right|<\infty$ for all $s \in[0,1)$. Returning to (15) we see that the sum in second term in (15) is $\sum_{k=0}^{n-1} \frac{\Lambda(1-s)}{\Lambda(1-s) \nu k+1}+\sum_{k=0}^{n-1} u_{k}(s)$. In turn by standard arguments [4, p. 544] we see that the expression

$$
\sum_{k=0}^{n-1} \frac{\Lambda(1-s)}{\Lambda(1-s) \nu k+1}-\frac{\ln (1+\nu n \Lambda(1-s))}{\nu}
$$

is bounded uniformly for $s \in[0,1)$ and approaches a limit as $n \rightarrow \infty$ which is a bounded function of $s \in[0,1)$. Thus since the bound on the $u_{k}(s)$ is uniform for $s \in[0,1)$, the expression

$$
\frac{1}{\Lambda\left(R_{n}(s)\right)}-\frac{1}{\Lambda(1-s)}-\nu n-\frac{1+\nu}{2} \cdot \ln (1+\nu n \Lambda(1-s))-\sum_{k=0}^{n-1} \gamma_{k}(s)
$$

converges to a bounded limit as $n \rightarrow \infty$ uniformly for $s \in[0,1)$. Finally, since the second sum in (15) $\sum_{k=0}^{n-1} \gamma_{k}(s)=o(\ln n)$ the formula (14) is fair. The lemma is proved.

Remark 1. The assertion (14) was proved in [5, pp. 20-21] provided that $F^{\prime \prime \prime}(1-)$ is finite.

Now using Lemma 2.3 we can improve the assertion (2). In fact putting $s=0$ and, after elementary arguments we obtain the following results.

Theorem 2.1. Let conditions (1), (4) and (7) hold. Then

$$
\mathbb{P}\{\mathcal{H}>n\}=\frac{\mathcal{N}(n)}{(\nu n)^{1 / \nu}}\left(1-\frac{1+\nu}{2 \nu^{2}} \frac{\ln n}{n}+o\left(\frac{\ln n}{n}\right)\right),
$$

as $n \rightarrow \infty$, where $\mathcal{N}(n)$ is $S V$-function and defined in Lemma 1.1

By the same way we obtain the following local limit theorem which is improvement of the analogous result from the paper [6].

Theorem 2.2. If conditions (1), (4) and (7) hold, then

$$
(\nu n)^{1+1 / \nu} \cdot P_{11}(n)=\frac{\mathcal{N}_{\nu}(n)}{p_{0}}\left(1-\frac{(1+\nu)^{2}}{2 \nu^{2}} \frac{\ln n}{n}+o\left(\frac{\ln n}{n}\right)\right),
$$

where $\mathcal{N}_{\nu}(n) \mathcal{N}^{-1}(n) \rightarrow 1$ as $n \rightarrow \infty$.

\section{References}

[1] S.Asmussen, H.Hering, Branching processes, Birkhäuser, Boston, 1983.

[2] K.B.Athreya, P.E.Ney, Branching processes, Springer, New York, 1972.

[3] N.H.Bingham, C.M.Goldie, J.L.Teugels, Regular Variation, Univ. Press, Cambridge, 1987. 
[4] G.M.Fihtengols, Cours of differential and integral calculus, vol. 2, Nauka, Moscow, 1970 (in Russian).

[5] T.E.Harris, The theory of branching processes, Springer-Verlag, Berlin, 1963.

[6] A.A.Imomov, On a limit structure of the Galton-Watson branching processes with regularly varying generating functions, Probability and mathematical statistics (to appear), 2018.

[7] J.Karamata, Sur un mode de croissance reguliere. Theoremes fondamenteaux, Bull. Soc. Math. France, 61(1933), 55-62.

[8] J.Karamata, Sur un mode de croissance réguliére des fonctions, Mathematica (Cluj), 4(1930), 38-53.

[9] E.Seneta, Regularly varying functions in the theory of simple branching process, Adv. Appl. Prob., 6(1974), 408-420.

[10] E.Seneta, A Tauberian Theorem of E.Landau and W.Feller, Ann. Prob., 1(1973), 1057-1058.

[11] E. Seneta, Regularly Varying Functions, Springer, Berlin, 1972.

[12] E.Seneta, On invariant measures for simple branching process, Jour. Appl. Prob., 8(1)(1971), 43-51.

[13] R.S.Slack, Further notes on branching processes with mean 1, Wahrscheinlichkeitstheor. und Verv. Geb., 25(1972), 31-38.

[14] R.S.Slack, A branching process with mean one and possible infinite variance, Wahrscheinlichkeitstheor. und Verv. Geb., 9(1968), 139-145.

[15] V.M.Zolotarev, More exact statements of several theorems in the theory of branching processes, Theory Prob. and Appl., 2(1957), 245-253.

\title{
О применении медленно меняющихся функций с остатком в теории ветвящихся процессов Гальтона-Ватсона
}

\author{
Азам А. Имомов \\ Государственный центр тестирования при Кабинете Министров Республики Узбекистан \\ Богишамол, 12, 100202, Ташкент \\ Каршинский государственный университет \\ Кучабаг, 17, Карши, 180100, Узбекистан \\ Эркин Э. Тухтаев \\ Каршинский государственный университет \\ Кучабаг, 17, Карши, 180100, Узбекистан

\footnotetext{
В работе мы исследуем применение медленно меняющихся функций (в смысле Карамата) в теории ветвлщихся прочессов Гальтона-Ватсона. Рассмотрим критический случай такой, что производящая функиия распределения прямого потомка одной частицы имеет бесконечный второй момент, но его хвост регулярно меняется с остатком. Мы уточняем основную лемму теории критических ветвящихся процессов Гальтона-Ватсона и улучшаем некоторые известные асимптотические результаты.
}

Ключевые слова: ветвящийся прочесс Гальтона-Ватсона, медленно меняющиеся функиии, производящие функиии. 